\title{
Cuidados paliativos: o valor da pessoa e sua história no HUPE
}

\author{
Lilian Hennemann-Krause, ${ }^{1 *}$ Janete A. Araújo, ${ }^{1}$ Danielle de M. Florentino, ${ }^{2}$ Elisabeth M. Petersen ${ }^{2}$
}

\begin{abstract}
Resumo
O Núcleo de Cuidados Paliativos (NCP) atua no Hospital Universitário Pedro Ernesto (HUPE) desde 2009, prestando assistência continuada aos pacientes com câncer oriundos dos diversos serviços do hospital. O modelo de cuidadoé multiprofissional, centrado na pessoa e no familiar (cuidador), focando na excelência técnica e humanista do controle de sintomas físicos, emocionais, sociais e espirituais. Foram atendidos 903 pacientes desde então. As atuações dos profissionais da equipe médica, psicóloga, fisioterapeuta e musicoterapeuta são relatadas de acordo com seus olhares e competências na prática diária do Núcleo de Cuidados Paliativos (NCP-HUPE). Na história do NCP são apresentados os indicadores de assistência e atuações dos profissionais relacionados à assistência, ensino e pesquisa. A assistência do NCP tem favorecido que pacientes e familiares possam viver bem até o fim, com dignidade, conforto e controle dos sintomas. Considera-se uma necessidade urgente incluir a paliação na prestação de cuidados continuados nos sistemas de saúde, de modo a favorecer o acesso equitativo aos cuidados de saúde de forma integrada à rede de assistência em todos os níveis: assistência primária, secundária e terciária, unidades de urgência/emergência, instituições de internação de longa permanência, visando o ideal de manter serviços de qualidade para atender necessidades dos pacientes e de suas famílias. Atenção especial deve ser dada à adequada formação de profissionais de saúde em cuidados paliativos e no controle da dor, de modo a garantir acesso à assistência de qualidade, além de ensino e pesquisa.
\end{abstract}

Descritores: Cuidados paliativos; Equipe de assistência ao paciente; Indicadores básicos de saúde; Avaliação de sintomas; Manejo da dor; História.

\section{Abstract \\ Palliative Care: the person's value and its history in HUPE}

The Núcleo de Cuidados Paliativos (NCP) (Nucleus of Palliative Care) began its work at the Hospital Universitário Pedro Ernesto in 2009, providing ongoing care to cancer patients referred from other services within the hospital. The model of care is multidisciplinary, person and family (caregiver) centered, focusing on technical excellence and humanistic approaches to control symptoms and tend to physical, emotional, social, and spiritual needs. In this period 903 patients were assisted. The performance of the professionals on the team - physician, psychologist, physical therapist, and music therapist - is presented according to their own perspectives and skills in daily practice. This NCP history includes assistance
1. Núcleo de Cuidados Paliativos. Hospital Universitário Pedro Ernesto. Universidade do Estado do Rio de Janeiro. Rio de Janeiro, RJ, Brasil.

2. Capacitação em Cuidados Paliativos. Núcleo de Cuidados Paliativos. Hospital Universitário Pedro Ernesto. Universidade do Estado do Rio de Janeiro. Rio de Janeiro, RJ, Brasil.

\section{*Endereço para correspondência:}

Hospital Clementino Fraga Filho, UFRJ

Rua Itacuruça, 60/501

Rio de Janeiro, RJ, Brasil. CEP: 20510-150.

E-mail: hennemann33@gmail.com

Revista HUPE, Rio de Janeiro, 2015;14(Supl. 1):19-27 doi:10.12957/rhupe.2015.17778

Recebido em 03/06/2015. Aprovado em 15/07/2015.

indicators and the role of each professional in assistance, education, and research. The NCP has enabled patients and families to live with quality of life, with dignity, comfort, and symptom control until the end. It's urgent to include palliation in the ongoing care in order to promote equitable access to quality healthcare and assistance to patients and their families in all levels: primary, secondary, and tertiary care, including emergency units and long-term care institutions. Special attention should be given to appropriate training of health professionals in palliative care and pain control to ensure good assistance, education and research.

Keywords: Palliative care; Patient care team; Health status indicators; Symptom assessment; Pain management; History.

\section{Resumen}

\section{Cuidados Paliativos: el valor de la persona y su historia en HUPE}

El Núcleo de Cuidados Paliativos (NCP) actúa en el Hospital Universitario Pedro Ernesto (HUPE) desde 2009, proporcionando continuidad en la atención a pacientes con cáncer encaminados de los distintos servicios del hospital. El modelo de atención es multidisciplinar, focalizado en la persona y la familia (cuidador), se centra en la excelencia técnica y humanista de control de síntomas físicos, emocionales, sociales y espirituales. En este período fueron atendidos 903 pacientes. Se presenta, de acuerdo con su perspectiva y habilidades en la práctica diaria del Núcleo de Cuidados Paliativos (NCP-HUPE), la actuación de profesionales del equipo: médico, psicólogo, fisioterapeuta y musicoterapeuta. En la historia del NCP se muestran los indicadores de asistencia y las actuaciones de los 
profesionales relacionados con la educación y la investigación. El NCP ha favorecido para que los pacientes y las familias puedan vivir con calidad de vida, dignidad, confort y control de los síntomas hasta el final. Se considera el carácter de urgencia para su inclusión de los cuidados paliativos a los cuidados continuos en los sistemas de salud, con el fin de fomentar el acceso equitativo a todos los niveles de asistencia, unidades de emergencia, instituciones de atención continuada, buscando

\section{Introdução}

O impacto sobre o controle da dor no câncer tem uma repercussão tão importante que tornou patente que, além da dor física, outras dimensões do sofrimento humano deveriam ser abordadas, conforme Cicely Saunders já enfatizara duas décadas antes sobre a dor total. ${ }^{1} \mathrm{O}$ controle da dor física possibilita a expressão dos sentimentos e emoções, a convivência familiar e reflexões sobre o sentido e significado da vida.

Em 1990, a Organização Mundial de Saúde (OMS) lançou a definição sobre o que são cuidados paliativos relacionados ao câncer e o que cabe a esta abordagem. ${ }^{2}$ Em 1998, definiu cuidados paliativos para crianças, reforçando a sua necessidade em crianças de diferentes idades e o apoio que as famílias devem receber.

Em 2002, o conceito de cuidados paliativos foi revisado, sendo incluídas outras doenças crônicas que ameaçam a vida da mesma maneira que o câncer. A orientação foi direcionada para uma abordagem conjunta com outros tratamentos, sugerindo que os cuidados paliativos fossem iniciados junto com as terapias de cura ou modificadoras do curso da doença, em caso de ameaça à vida. Além disto, manifestou-se a preocupação em cuidar do luto dos familiares e cuidadores. ${ }^{3}$ Atualmente, a OMS define cuidados paliativos como: uma abordagem que melhora a qualidade de vida do paciente e de suas famílias ajudando-os a lidar com os problemas associados às doenças ameaçadoras à vida, prevenindo e aliviando o sofrimento através da identificação precoce, impecável avaliação e tratamento da dor e outros problemas, físicos, psicossociais e espirituais.

Sendo os princípios norteadores dos cuidados paliativos:

a) promover o alívio da dor e de outros sintomas angustiantes;

b) ratificar a vida e considerar a morte como um processo natural;

c) nem acelerar nem adiar a morte; mantener un servicio de calidad para satisfacer las necesidades de los pacientes y sus familias. Especial atención se debe dar a la formación adecuada de los profesionales de salud en cuidados paliativos y control del dolor, para garantizar el acceso a una buena asistencia, educación e investigación.

Palabras clave: Cuidados paliativos; Grupo de atención al paciente; Indicadores de salud; Evaluación de síntomas; Manejo del dolor; Historia.

d) integrar os aspectos psicológicos e espirituais do cuidado;

e) oferecer um sistema de apoio para ajudar os pacientes a viverem tão ativamente quanto possível até a morte;

f) oferecer um sistema de apoio para ajudar a família a enfrentar o período de doença do paciente e no seu próprio luto;

g) usar uma abordagem de equipe para acessar as necessidades dos pacientes e suas famílias, incluindo aconselhamento de luto, se indicado;

h) realçar a qualidade de vida, que pode influir positivamente no curso da doença;

i) aplicá-la precocemente no curso da doença, em conjunto com outras terapias que pretendem prolongar a vida, como quimioterapia ou radioterapia, e incluir as investigações necessárias para melhor compreensão e tratamento de complicações clínicas angustiantes. ${ }^{4}$

Evidentemente, esta definição não estabelece o tempo de vida estimado para os cuidados paliativos, porém deixa implícito o cuidado integral da pessoa. Também não especifica o modelo de atenção (assistência ambulatorial, domiciliar ou hospitalar; internação de curta ou de longa permanência), mas a filosofia de cuidado.

Os cuidados paliativos apresentam um modelo inovador e singular para as políticas de saúde e sociais, pois focam na autonomia do paciente e usam uma abordagem integral que combina excelência técnica e conhecimento (ex.: controle de sintomas), habilidades (ex.: comunicação) e atitudes (ex.: encarar cada paciente como um indivíduo com uma rica história de vida).

\section{Materiais e métodos}

O trabalho tem natureza qualitativa, utilizando a descrição da atuação do médico, da psicóloga, da fisioterapeuta e da musicoterapeuta de acordo com seus olhares e competências na prática diária de atuação do NCP do HUPE. No relato da trajetória do NCP são 
apresentados os indicadores de assistência e atuações dos profissionais relacionadas ao ensino e pesquisa realizados desde o início da assistência específica em 2009 até hoje.

\section{Medicina paliativa}

A medicina sempre teve como finalidade cuidar dos pacientes, independentemente da fase de doença. Ela sempre fez isto e sempre fará. Talvez não seja muito claro para todos, mas quando se faz a opção por ser um profissional de saúde se opta por assistir o sofrimento humano e por atuar ativamente para aliviá-lo através da cura, cuidado e conforto.

Atualmente, há uma dificuldade em nomear o cuidado na fase final da vida e definir o significado de "cuidados paliativos".É visível a dificuldade dos profissionais, principalmente dos mais novos, em abordar os temas relacionados ao sofrimento, fim de vida e morte, seja devido aos valores da sociedade atual pontuados pela capacidade de produção, pela dificuldade de discutir o assunto, ou pela deficiência do ensino sobre o tema, seja pelos aspectos éticos profissionais. Afinal, lidar com a finitude humana é também lidar com os nossos próprios limites e com os limites que a vida nos impõe. A finitude humana nos faz refletir sobre os limites das intervenções tecnológicas, não obstante serem cada vez melhores e maiores. Não é possível afastar a morte indefinidamente. Por outrolado, também não podemos enfrentar a morte como um inimigo a ser combatido a qualquer custo, pois o sangue no campo de batalha é o mesmo, seja dos vencidos, seja dos vencedores. Caso a morte seja encarda desta maneira, cada vez que um paciente morrer, seja qual for a idade, o profissional vai tender a se sentir derrotado ou fracassado. Há que se aceitar que a morte faz parte do processo natural da vida, tão cheia de mistérios, honra e dignidade como o próprio nascimento.

Sem dúvidas, a vida física é um bem em si mesma e de caráter inviolável, que delineia a condição essencial para o exercício das diversas outras dimensões da vida humana. A fragilidade que advém da doença exige uma atitude humana e técnica que é cuidar do outro, especialmente dos que sofrem, incluindo seus familiares. A medicina paliativa é um tipo de assistência especializada que não tem menos valor porque não "salva vidas". ${ }^{5}$ A medicina paliativa atua de modo igualmente importante: favorece que a pessoa viva o final de sua vida terrena plenamente e com toda dignidade.

Todas as pessoas têm a mesma dignidade, pois não há um ser humano mais digno do que outro. É preciso cuidar da pessoa até o fim de sua vida, seja recém-nascido ou muito velho, independentemente da doença que a acomete, da classe social, da história de vida.é preciso que se apoie este ser humano a escrever o último capítulo do seu livro da vida, a fim de que ele possa fechar este livro com honra e dignidade. Quando não é mais possível controlar a doença, previne-se o sofrimento evitável, tratando a dor e outros sintomas biopsicossociais e espirituais de forma impecável, com os recursos técnico-científicos disponíveis e também humanistas.

A atuação em cuidados paliativos nos lembra da transitoriedade da vida e que devemos viver bem cada dia, principalmente hoje, justamente porque pode ser a véspera de uma grande jornada.

\section{A prática do psicólogo em equipe de cuidados paliativos}

Na prática do profissional de psicologia, compondo a equipe multiprofissional do Núcleo de Cuidados Paliativos, duas vertentes são importantes em relação à sua atuação: a equipe e os pacientes.

Uma das atuações importantes é cuidar da equipe, valorizando o trabalho dos seus membros e favorecendo a comunicação verdadeira e respeitosa, intermediando atritos entre as diversas personalidades. O psicólogo éo grande facilitador da comunicação, elemento essencial, pois através de uma boa comunicação saberes são trocados, constroem-se conhecimentos, amenizam-se expectativas e frustrações da equipe.

Em relação à abordagem dos pacientes, é preciso observar os conteúdos emocionais envolvidos no relato das queixas trazidas pelo paciente e seus sintomas. Devese perceber a figura do paciente dentro de um contexto, a fim de se oferecer a atenção integral e a identificação de desordens psíquicas que geram sofrimento, bem como dos mecanismos de defesa negativos; assim, pode-se ajudar na reorganização da vivência de doença e no uso de recursos adaptativos para manter o paciente participativo no processo de tratamento. ${ }^{6}$

A construção de um vínculo de confiança, através de escuta acolhedora verbal e não verbal, empatia e respeito, além das questões técnicas e humanísticas favorecem a questão da morte como um processo natural. As fantasias acerca da morte, do morrer e do desejo de imortalidade são o ponto primordial para a ressignificação da intensa experiência que é o processo de terminalidade da vida, com especial atenção à linguagem simbólica e ao não dito.? 


\section{Artigo original}

É fundamental perceber o fenômeno religioso do paciente e sua família como um recurso que possibilita buscar alternativas para reforçar o seu suporte emocional, facilitando a busca pelo sentido à vida e do sofrimento humano presente no processo de adoecimento. ${ }^{8}$ Através das crenças trazidas pelo paciente, pode-se levar em conta e pontuar o que é significativo para o mesmo.

É importante oferecer um suporte à família para facilitar a compreensão do processo de adoecimento em todas as suas fases, visando diminuir o impacto sentido por eles que estão também vivenciando, muitas vezes, reações emocionais de angústia, medo, culpa. ${ }^{9}$ Segundo Fonseca, além de a família sofrer um impacto pela doença de um dos seus entes queridos, ela necessita manter o equilíbrio para poder assegurar o cumprimento das tarefas e das necessidades do paciente..$^{10}$ É importante perceber a redistribuição dos papéis e responsabilidades; e a partir daí a adaptação à ausência futura e às perdas a serem enfrentadas. Durante todo o processo de acompanhamento do paciente e da família deve-se facilitar a expressão dos sentimentos e emoções.

O psicólogo também pode ser o elemento facilitador do exercício da autonomia do paciente, alicerçado num modelo bioético de apoiar as suas próprias escolhas, intermediando esse processo junto a equipe e família. Este apoio pode ocorrer em situações de reintegração do paciente à sociedade e à rotina familiar, buscando, junto com o paciente e familiares, as melhores formas de adaptação às limitações impostas pela doença, evitando o surgimento de complicações de ordem psicológica que possam interferir no campo profissional, afetivo e social tanto do sujeito em tratamento quanto de seus familiares. ${ }^{11}$

Para o exercício da autonomia, é essencial que se forneça informações claras sobre a doença, sua evolução e procedimentos, respeitando seus limites de compreensão e tolerância emocional. As decisões fundamentais geralmente sobre tomada de decisões antecipadas de fim de vida devem ser discutidas com o doente ou seu representante legal, sendo respeitada sua vontade. ${ }^{2}$

Por fim, mas não menos importante, o psicólogo oferece acompanhamento de apoio e suporte aos familiares após o óbito do paciente, "permitindo e autorizando" o sofrimento, de forma a favorecer um processo de luto considerado normal, procurando prevenir a evolução de um luto complicado.

Portanto, o psicólogo em uma equipe de cuidados paliativos atua na busca de estratégias para ajudar o paciente e familiares/cuidadores no enfrentamento e elaboração das experiências emocionais vivenciadas na fase final da vida. Ele precisa ter cuidado para não ocupar o lugar de um elemento invasivo no processo de adoecimento, mas, sim, de ser um facilitador que favorece a integração do paciente, da família e da equipe multidisciplinar, mantendo o foco na pessoa (e não na doença) e na melhoria da qualidade de vida do paciente, da família e dos cuidadores. O momento vivido pode ser compartilhado através da busca por recursos internos, atenuando sentimentos de solidão, culpa, fracasso e possibilitando o alívio do sofrimento. Pode e deve ser um momento de crescimento pessoal para todos.

\section{Fisioterapia paliativa: reflexões e ações}

A atuação da fisioterapia em cuidados paliativos possibilita ao paciente o enfrentamento de uma doença avançada proporcionando qualidade de vida, através de condutas que o mantenham funcional em suas práticas diárias. A reabilitação frente a uma enfermidade requer estratégias de prevenção, restauro de suporte e de paliação. ${ }^{13}$

A fisioterapia oferece um suporte para que os pacientes vivam o mais ativamente possível, com dignidade e conforto, além de oferecer ajuda à família na assistência ao paciente, no enfrentamento da doença e luto. ${ }^{14,15,16} \mathrm{O}$ fisioterapeuta possui métodos e técnicas que são imensamente úteis na abordagem dos cuidados paliativos. Porém, é necessário que esteja preparado, técnica e emocionalmente, a fim de proporcionar o adequado atendimento. ${ }^{17}$

A atuação do fisioterapeuta deve ser consoante à funcionalidade do paciente, ou seja, quando totalmente dependente, o enfoque deve ser nos cuidados ativos de conforto, como o posicionamento e orientação quanto às mudanças de decúbito, transferências e mobilização global, prevenindo deformidades e outras complicações. Em casos de maior independência, estimular o autocuidado, atividades de vida diária, adequando o ambiente e favorecendo a funcionalidade e maximização da autonomia. 13,14,15

Neste contexto, o fisioterapeuta procura reduzir a dependência e dar apoio aos cuidadores e familiares, construindo uma educação e treinamento, atuando na prevenção da deterioração funcional, recuperação da autonomia pessoal e de alívio de outros sintomas. Desta forma, busca a manutenção e melhora da mobilidade articular, preservação, quando possível, da função muscular, prevenção e minimização dos efeitos da atrofia e das complicações respiratórias. ${ }^{13,16}$ 
A fisioterapia busca não somente a funcionalidade, mas também a coesão social, maximização do significado da vida e estabelecimento de estratégias de suporte. Os fisioterapeutas, pela sua própria intervenção, têm a oportunidade de ouvir, descobrir e discutir valores de vida e crenças do paciente, assim como as estratégias para gerenciar os seus sintomas e capacidades durante o dia. Na relação fisioterapeuta-paciente, o profissional pode criar oportunidades nas quais o paciente acaba por discutir suas preocupações, desgostos e reflexões relativas ao momento que está a viver, ganhando um conhecimento das ansiedades e angústias relacionadas com a perspectiva da morte do seu paciente. $\mathrm{O}$ fisioterapeuta contribui, desta forma, para a qualidade de vida do paciente em processo de morte, assistindo-o na reflexão dos propósitos, das prioridades e dos significados da sua vida. ${ }^{18}$

\section{Musicoterapia em cuidados paliativos na assistência ambulatorial}

Dentre as terapêuticas de cuidado oferecidas aos pacientes em cuidados paliativos atendidos no NCPHUPE, a musicoterapia foi a mais recente, tendo se integrado ao tratamento a partir de 2011.

Nesse percurso, as reflexões foram uma constante, tanto na construção de uma abordagem diferenciada no espaço ambulatorial, em condições extremamente especiais, quanto nos aspectos enfatizados como objetivos terapêuticos das intervenções.

A musicoterapia em cuidados paliativos pode ser definida como uma terapia não farmacológica, não invasiva, que utiliza o som e a música estruturada e seus elementos constituintes (ritmo, melodia, harmonia) em experiências musicais variadas, envolvendo, de forma inter-ativa, ${ }^{19}$ o conjunto pacientes-musicoterapeutafamiliares-cuidadores-profissionais.

A 'música viva', praticada no momento da consulta por esses atores, possibilita a adequação das propostas e dos elementos musicais às demandas apresentadas pelo paciente no instante presente.

A partir de uma avaliação inicial pelo musicoterapeuta se estabelece um continente sonoro seguro para a expressão de pensamentos, sentimentos e emoções relacionados a: quadro clínico, vivência do agravamento, progressão da doença, suas consequências físicas, psicossociais e espirituais intensificadas nessa fase, luto antecipatório dos familiares e a perspectiva da morte. ${ }^{20}$

$\mathrm{O}$ aspecto lúdico e prazeroso da música provoca a estimulação de conexões cerebrais deáreas responsáveis pela evocação de memórias de situações vivenciadas em várias épocas da existência, envolvendo redes de relacionamentos e afetos que ajudam a encontrar um sentido para a vida de cada paciente e para o sofrimento presente. ${ }^{21}$ Essas reminiscências promovem um feedback emocional positivo e qualidade de vida.

Nesse contexto, a lembrança de um repertório familiar oferece uma nova perspectiva de conhecimento da história do sujeito, que é o foco do nosso cuidado, utilizando um viés até certo ponto inesperado para paciente e familiares, no setting hospitalar: a produção musical. É dada ênfase à valorização do que ainda é saudável e criativo no paciente através do canto e do acompanhamento instrumental das músicas preferidas do paciente, sejam elas populares, folclóricas/regionais, eruditas ou religiosas. A comunicação revestida de caráter estético que se processa com essa "trilha sonora"22 contribui para o resgate de uma identidade singular do paciente que precedia a doença para o compartilhamento com o grupo familiar e para o despertar de um sentimento de pertencimento; ${ }^{23}$ além disso, resulta em aumento da autoestima, melhoria de parâmetros clínicos, como dor, dispneia, fadiga depressão e ansiedade, maior bem-estar, reafirmação de crenças religiosas e vivência da espiritualidade numa vinculação com o sagrado e a transcendência.

Numa outra vertente de intervenção, as improvisações destacam-se como o elemento-surpresa, criando a oportunidade ao paciente de lançar-se numa nova perspectiva de produção musical: de "ter respostas individuais criativas frente ao adoecimento e de aprender a lidar com as próprias reações emocionais nas atuais contingências da vida". ${ }^{24}$

Com uma atuação dinâmica, mas respeitando as limitações impostas pela progressão da doença e a condição clínica apresentada a cada consulta, o investimento está sempre orientado para a competência e o potencial máximo do paciente nas ações e tomada de decisões, resultando em empoderamento e manutenção de um protagonismo. Em muitas situações da prática clínica, a interdisciplinaridade favorece a atuação da musicoterapia com outras abordagens, trazendo maior benefício para o paciente com a intervenção conjunta. Incluem-se aqui as propostas de acompanhamento aos familiares na elaboração do luto ou de rituais de despedida.

A característica da música de ultrapassar barreiras e transcender o tempo e o espaço revela-se também transformadora da própria ecologia do ambiente, das relações interprofissionais e da globalidade da ação no NCP. 


\section{Artigo original}

Pacientes e familiares tornam-se, na maioria das vezes, mais participantes, mais seguros nas manifestações de emoções, risos, de cantos e danças, do desejo de comemorar datas festivas com a equipe, ou mesmo de promover despedidas que sensibilizam a todos.

A prática clínica da musicoterapia no NCP vem corroborar o pensamento de Aldridge a respeito da composição das equipes de profissionais de cuidados paliativos, na qual a musicoterapia tem como objetivo maior: Procurar encorajar os pacientes a manterem algum bem-estar em face da iminente perda biológica e social (...) pois, [com a potência da música] e mesmo em meio ao sofrimento, é possível criar coisas belas. ${ }^{24}$

\section{O Núcleo de Cuidados Paliativos no Hospital Universitário Pedro Ernesto}

O NCP nasceu de uma ideia que paulatinamente foi se concretizando desde 2007, quando vários professores do HUPE estruturaram o projeto-piloto Associação da tecnologia da informação e da assistência interdisciplinar domiciliar aos pacientes portadores de doença neoplásica em fase avançada do HUPE - Centro Universitário de Controle do Câncer (CUCC). Neste projeto-piloto estava incluído um subprojeto de efetivação do Núcleo de Medicina Paliativa, com o seguinte planejamento:

Visa implantar um núcleo multidisciplinar de cuidados paliativos voltado para os pacientes com câncer avançado, integrado sob a ótica da dignidade do morrer. Tem como um dos objetivos apoiar o HUPE, retirando do corpo do hospital geral pacientes que necessitam de um enfoque diferenciado. Encontra-se em fase de elaboração do subprojeto definição de espaço físico para efeitos de instalação provisória, formação da equipe de saúde, bem como captação de recursos para a construção de suas instalações. Sua previsão é para o núcleo ocupar o $2^{\circ}$ andar (do CUCC) e posteriormente, já contemplando as internações, nos $4^{\circ}$ e $5^{\circ}$ andares.

O NCP iniciou suas atividades efetivamente com a vinda de uma médica paliativista para compor seu quadro em janeiro de 2009. O HUPE, habilitado como Unidade de Assistência de Alta Complexidade em Oncologia (UNACON), com serviços de radioterapia e hematologia), ${ }^{25}$ passou a seguir as diretrizes da Política Nacional de Atenção Oncológica, ${ }^{26}$ norma então vigente, oferecendo os cuidados paliativos àqueles usuários que necessitassem desta abordagem, através de uma equipe multiprofissional. A equipe, por sua vez, interagia com o corpo clínico hospitalar.

Os atendimentos tiveram início em 12 de janeiro de 2009 e, atualmente, ocorrem no prédio dos ambulatórios, sala 3, subdividida em cinco consultórios e utilizada duas vezes por semana em um turno diário.

A equipe multidisciplinar do Núcleo de Cuidados Paliativos, neste curto período de existência, tem sido composta por vários profissionais com diferentes vínculos e em momentos distintos: médicos, enfermeiros, nutricionista, psicólogas, fisioterapeuta, fonoaudióloga, assistentes sociais, odontólogo, musicoterapeuta, técnicos de enfermagem e técnico-administrativo.

Na área da educação, com o objetivo de divulgar o conhecimento específico da área, foram realizados três simpósios anuais - Simpósio de Cuidados PaliATIVOS do HUPE em 2010, ${ }^{27} 2011,{ }^{28}$ e 2012, com carga horária de 18 horas e cerca de 80 participantes inscritos em cada um deles. Todos os eventos foram gravados pelo Telessaúde UERJ e estão disponíveis no site em áudio e vídeo na íntegra. ${ }^{29} \mathrm{Em} 2010$, foram promovidos dois cursos de 10 horas cada, através da Coordenadoria de Desenvolvimento Acadêmico (CDA), para residentes da Clínica Médica do HUPE e da Medicina de Família e Comunidade do HUPE/UERJ e da Universidade Federal do Rio de Janeiro (UFRJ). Isso favoreceu uma maior divulgação do trabalho realizado pela equipe do NCP e integração com outros serviços do HUPE, além do objetivo específico de ensino e capacitação.

O NCP tem sido área de estágio, treinamento e prática para residentes, alunos ou estagiários de medicina, nutrição, psicologia, fisioterapia e fonoaudiologia.

A Faculdade de Ciências Médicas da UERJ, SubReitoria de Extensão, através da Psicologia Médica, vem realizando o primeiro curso de extensão, nível aperfeiçoamento teórico-prático, Controle de Sintomas em Cuidados Paliativos Oncológicos, no período de 27 de novembro de 2014 a 30 de julho de 2015, com duração total de 200 horas. O curso é multiprofissional e estão concluindo 32 profissionais de várias áreas de atuação.

A educação continuada da equipe vem sendo estimulada através da participação em cursos e congressos. A pesquisa foi desenvolvida, com aceitação para publicação de trabalhos em congressos de caráter nacional e internacional sobre cuidados paliativos nas várias especialidades e disciplinas. A Revista HUPE do segundo bimestre de 2012 foi dedicada aos cuidados paliativos e escrita pela equipe que apresentou sua experiência na área. ${ }^{30}$ 
O número de pacientes assistidos vem aumentando a cada ano. $O$ atendimento envolve a complexidade da excelência técnica, aliada ao humanismo e à ética bem sedimentada. A assistência não é dirigida apenas ao paciente, mas ao paciente-família (cuidador). Esta unidade de cuidado complexa é mais permeável à abordagem dos aspectos biopsicossociais e espirituais e torna o cuidado mais eficaz, com reflexo na qualidade de vida de todos os participantes. As consultas médicas duram de 30 a 40 minutos e podem envolver vários procedimentos - administração de medicamentos, hidratação subcutânea, curativos, paracentese, entre outros. As consultas são mensais, enquanto o quadro clínico do paciente permanecer estável. Existindo agravamento, o retorno passa a ser semanal, além de serem necessárias orientações a distância, por telefone, através do cuidador que comparece à consulta ou pelo Telesaúde em caso de haver disponibilidade e acesso. Em média, são feitos cerca de dez atendimentos a pacientes/familiares pela equipe por dia/turno.

O NCP tem também por objetivo apoiar outras clínicas do HUPE e, portanto, não atende por demanda espontânea, somente com encaminhamento interno. Cerca de 60\% dos encaminhamentos são oriundos da Oncologia Clínica, Cirurgia Torácica e Cirurgia de Cabeça e Pescoço. Quanto ao perfil dos pacientes do NCP, a prevalência é ligeiramente maior de homens (54\%), com a idade média de 64 anos. A grande maioria dos pacientes recebe cuidados de familiares e cerca de $66 \%$ residem no município do Rio de Janeiro [destes apenas 9\% moram na área programática do HUPE (AP 2.2)], e os outros 33\% residem em outros municípios do Estado do Rio de Janeiro, predominantemente da Baixada Fluminense. Todos têm diagnóstico de câncer avançado e retornam periodicamente para o controle de sintomas. As sedes dos tumores primários mais prevalentes são: pulmão, cabeça e pescoço e colorretal. O tempo de acompanhamento desde a admissão no NCP até o óbito vem progressivamente aumentando: em 2009 foi de 60 dias; em 2010, de 96 dias; em 2011 de 130 dias; e em 2012 de 138 dias. Isto ocorre devido ao encaminhamento mais precoce ou mesmo ao melhor controle dos sintomas e agravos clínicos. Aproximadamente 75\% dos óbitos são hospitalares (cerca de 30\% destes no HUPE) e o restante ocorre no domicílio. O NCP atendeu 903 pacientes de 2009 até maio de $2015 .{ }^{31}$

A dispensação de analgésicos opioides (morfina e metadona) para controle da dor é feita pela farmácia hospitalar do HUPE, conforme a prescrição médica que tem por base o Protocolo Clínico e Diretrizes
Terapêuticas da Dor Crônica do Ministério da Saúde. ${ }^{32}$ As medicações são disponibilizadas na forma farmacêutica de comprimidos, na quantidade necessária, até o retorno do paciente ou para, no máximo, trinta dias, conforme Portaria 344/98, da Agência Nacional de Vigilância Sanitária (ANVISA). ${ }^{33} \mathrm{O}$ tratamento com os opioides disponíveis é iniciado tão prontamente quanto o paciente tenha indicação (dor de moderada a forte intensidade) e mesmo que o paciente esteja em vigência de quimioterapia ou radioterapia.

Conforme estudo feito pelo NCP referente à intensidade da dor e analgesia oferecida a 216 pacientes atendidos em 2011, observou-se uma prevalência de dor relacionada ao câncer em $89 \%$ dos pacientes. Apenas $11 \%(n=24)$ dos pacientes não necessitaram fazer uso de analgésicos. A dor leve foi tratada com analgésicos comuns (dipirona, paracetamol e AINES) em 25\% ( $\mathrm{n}=$ 54) dos pacientes. Para a dor moderada foram prescritos opioides fracos (codeína e tramadol) em 13\% $(n=28)$ dos casos, e para a dor de forte intensidade os opioides fortes (morfina e metadona) em 51\% $(n=110){ }^{34}$

\section{Discussão}

O alívio da dor é considerado um direito humano, 35 pois nenhum ser humano deve receber tratamento desumano, degradante e cruel, conforme artigo $5^{\circ}$ da Declaração Universal dos Direitos Humanos. ${ }^{36}$ No Brasil, é reconhecido o direito ao tratamento da dor através de lei federal ${ }^{37}$ e portarias do Ministério da Saúde. ${ }^{38,39}$

O modelo de assistência do NCP é basicamente centrado no atendimento ambulatorial. Vários casos de urgência são resolvidos no próprio ambulatório; por exemplo reidratação, administração de medicamentos e analgésicos, paracentese, além de procedimentos, como curativos, orientação dos pacientes/familiares no cuidado de feridas e ostomias. Pareceres aos pacientes internados nas enfermarias e no plantão geral do HUPE compõem a rotina de trabalho. Também a assistência telefônica é um meio rápido e simples de comunicação que favorece orientação e conforto. A telemedicina pode ser outro recurso utilizado, pois aumenta o vínculo e gera confiança, com a assistência intensiva. A interação através de relatórios (cartas) com equipes da assistência primária (medicina de família) da área dos pacientes amplia as possibilidades do cuidado. A visita domiciliar, para ser implantada como atuação de rotina, necessita de uma equipe específica para esta finalidade, que deverá estabelecer critérios quanto à área programática de abrangência, critérios clínicos e 


\section{Artigo original}

socioeconômicos específicos.

Cicely Saunders, refletindo sobre o modelo de cuidados paliativos a ser implantado, orientava que se deveria "olhar em volta e observar o que tem sido feito, e então ver como as suas circunstâncias podem criar outra versão inovadora." ${ }^{\prime 1} \mathrm{O}$ Núcleo de Cuidados Paliativos tem favorecido que pacientes e familiares possam viver bem até o fim, com dignidade, alegria e controle dos sintomas.

\section{Conclusão}

É urgente a inclusão da paliação na prestação de cuidados continuados nos sistemas de saúde, uma vez que a integração inadequada dos cuidados paliativos é um fator que reduz o acesso equitativo aos cuidados de saúde. Também é essencial que haja comunicação e acesso à rede de assistência em todos os níveis - primária, secundária e terciária -, inclusive as unidades de urgência/emergência, instituições de cuidados continuados e de longa permanência, para que se possa manter a qualidade dos serviços e atender às necessidades dos pacientes e suas famílias. ${ }^{40}$

No âmbito do desenvolvimento de serviços, é fundamental que os profissionais de saúde recebam formação adequada sobre cuidados paliativos e controle da dor em níveis de graduação, pós-graduação, especialização e de educação continuada, seja de forma presencial e/ou por tele-educação. Assim será possível garantir acesso à assistência, ensino e pesquisa de qualidade a todos os segmentos envolvidos.

\section{Referências}

1. Saunders CM. The challenge of terminal care. In: Symington $T$, Carter RL, eds. Scientific Foundations of Oncology. London, England: Heinemann; 1976:673-679.

2. World Health Organization. Cancer Pain Relief and Palliative Care. Report of a WHO Expert Committee. Technical Report Series No 804, Geneva: World Health Organization; 1990.

3. Sepúlveda C, Marlin A, Yoshida T, et al. Palliative care: the World Health Organization's Global perspective. Journal of Pain and Sympton Management. 2002:24(2):91-6.

4. World Health Organization. Cancer. Palliative Care. Definition of Palliative Care - [atualizada em 2014; acesso em: 2014 mar 14]. Disponível em: <http://www.who.int/cancer/palliative/definition/en/>.

5. Papa Francisco. 21st Plenary Assembly of the Pontifical Academy for Life. Assistance to the elderly and palliative care, Vatican City, March 05, 2015. Disponível em: <http://www. zenit.org/en/articles/pope-francis-address-to-the-pontificalacademy-for-life>. Acesso em: May, 17, 2015.

6. Othero MB, Costa DG. Propostas desenvolvidas em cuidados paliativos em um hospital amparador - terapia ocupacional e psicologia. Revista prática Hospitalar. 2007 jul-ago;52:157-160.
7. Kovács MJ. Educação para a morte: temas e reflexões. São Paulo: Casa do Psicólogo; 2008.

8. Silva, KS da. Em defesa da sociedade: a invenção dos cuidados paliativos. [dissertação]. Porto Alegre: Universidade Federal do Rio Grande do Sul; 2010.

9. Menezes CNB, Passareli PM, Santos MA dos, et al. Câncer infantil: organização familiar e doença. Revista Mal-estar e Subjetividade, Fortaleza, 2007:VII(1):191-210.

10. Fonseca JP. Luto antecipatório. Campinas: Editora Livro Pleno; 2004.

11. Sampaio AS, Löhr SS. Atuação em casas de apoio: pensando o papel da psicologia e construindo novos caminhos. RUBS. 2008 set-dez;1(3):52-60.

12. Oliveira AC de: Silva MJP. Autonomia em cuidados paliativos: conceitos e percepções de uma equipe de saúde. Acta Paul Enferm, São Paulo. 2010;23(2):212-217.

13. Florentino $D$, et al. A fisioterapia no alívio da dor: uma visão reabilitadora em cuidados paliativos. Revista Hospital Universitário Pedro Ernesto, 2012:11.2. Disponível em: <http://revista. hupe.uerj.br/default.asp?ed=62>.

14. Arrais RCS. Atuação da fisioterapia nos cuidados paliativos oncológicos. Disponível em: <http://www.sotamig.com.br/ downloads/2014/_trab_03.pdf>.

15. Cruz HAG. Papel do fisioterapeuta nos cuidados paliativos. Dissertação de mestrado. 2015. Disponível em: <http://hdl. handle.net/10400.11/2752//>.

16. Vaz J. Papel do fisioterapeuta numa equipa de cuidados paliativos. Dissertação de mestrado. 2013. Disponível em: <http:// hdl.handle.net/10216/72712//>.

17. Rosario MAB, Fraile AA. Fundamentos y objetivos de los cuidados paliativos. Aten. Primaria; 2002.

18. Girão M, Alves S. Fisioterapia nos cuidados paliativos. Revista de Ciências da Saúde da ESSCVP; 5, 2013.

19. Barcellos LRM. A Movimentação musical em musicoterapia: interações e intervenções. Cadernos de musicoterapia no 1. Rio de Janeiro: Enelivros; 1992.

20. Petersen EM. Buscando novos sentidos à vida. Revista HUPE. Cuidados Paliativos. 2012;11. Disponível em: <http://revista. hupe.uerj.br/default.asp?ed=62>.

21. FrankI VE. Em busca de sentido. Petrópolis, BR: Vozes; 2003.

22. Ruud E. Music therapy: improvisation, communication and culture. Gilsum, NH: Barcelona Publishers; 1998.

23. Boyce-Tillman J. Consctructing musical healing: the wounds that sing. London: Jessica Kingsley Publishers. 2000; p.38.

24. Aldridge D. Life as jazz: hope, meaning and music therapy in the treatment of life-threatening illness. In: C. Dileo. (Ed.) Music therapy and medicine: theoretical and clinical applications Silver Spring, MD: The American Music Therapy Association, Inc. 1999. p.79-94.

25. Brasil. Ministério da Saúde. Portaria SAS/MS no 102, de 3 de fevereiro de 2012. Exclui da Tabela de Habilitações de Serviços Especializados do Sistema do Cadastro Nacional de Estabelecimentos de Saúde (SCNES), o código de habilitação 17.05 - Serviço Isolado de Quimioterapia. Brasília: Diário Oficial da União, 07 fev 2012. Seção 1, p.42.

26. Brasil. Ministério da Saúde. Portaria no 2.439/GM de 8 de dezembro de 2005. Institui a Política Nacional de Atenção Oncológica: Promoção, Prevenção, Diagnóstico, Reabilitação e Cuidados Paliativos, a ser implantada em todas as unidades federadas, respeitadas as competências das três esferas de gestão. Brasília: Diário Oficial da União, 9 dez 2005. Seção 1, p.80-81. 
Lilian Hennemann-Krause e cols. • Cuidados paliativos: o valor da pessoa e sua história no HUPE

27. Hospital Universitário Pedro Ernesto. Jornal do HUPE. 2010 set;3(28):3.

28. Hospital Universitário Pedro Ernesto. Jornal do HUPE. 2011 nov-dez;4(42):4.

29. Telessaúde UERJ. UERJ. HUPE. Simpósios de Cuidados Paliativos 2012. [atualizado em 2014; acesso em 2014 Mar 20]. Rio de Janeiro. Disponível em: <http://www.telessauderj.uerj. br/ava/course/view.php?id=307>.

30. Cuidados Paliativos. Revista HUPE. 2012 abr-jun;11(2). Disponível em: <http://revista.hupe.uerj.br/default.asp?ed=62> Acessado em: 23 jan 2014.

31. Livro de Registro de pacientes, Núcleo de Cuidados Paliativos, HUPE, UERJ.

32. Portaria no 1.083/SAS/MS, de 2 de outubro de 2012. Aprova o Protocolo Clínico e Diretrizes Terapêuticas da Dor Crônica. Brasília: Diário Oficial da União, 03 out. 2012. Seção 1, p.54.

33. Portaria no 344, ANVISA, de 12 de maio de 1998. Aprova o Regulamento Técnico sobre substâncias e medicamentos sujeitos a controle especial. Republicada por ter saído com incorreções do original republicado no Diário Oficial da União de 31 de dezembro de 1998, Seção I.

34. Hennemann-Krause L, Araújo JA, Florentino DM, et al. Estudo da prevalência e intensidade da dor no câncer através da prescrição orientada pela escada analgésica no Núcleo de Cuidados Paliativos. Temas livres/pôsteres do 10o Congresso Brasileiro de Dor; 2012 Sep 26-29; Salvador, BA. S. Paulo: Ed. Âmbito; 2012. p. 92.

35. European Federation of IASP Chapters. EFIC's declaration on pain as a major health problem, a disease in its own right, May 2001. Disponível em: <http://www.efic.org/about_pain.htm\#efic_declaration>. Acesso em: 29 jan 2014.

36. United Nations. The Universal Declaration of Human Rights, UN, 1948. Disponível em: <http://www.un.org/en/documents/ udhr/index.shtml>. Acesso em: 29 jan 2013.

37. Lei no 12.732. Presidência da República, de 22 de novembro de 2012, Brasília: Diário Oficial da União, 23 nov 2012, p.1.

38. Portaria no $876 \mathrm{MS} / \mathrm{GM}$, de 16 de maio de 2013. Brasília: Diário Oficial da União, 17 mai. 2013, Seção 1, p.135-136.

39. Portaria no 874 MS/GM, de 16 de maio de 2013, Política Nacional para a Prevenção e Controle do Câncer na Rede de Atenção à Saúde das Pessoas com Doenças Crônicas no âmbito do Sistema Único de Saúde (SUS). Brasília: Diário Oficial da União, 17 mai 2013, p.129.

40. World Health Organization. Strengthening of palliative care as a component of integrated treatment within the continuum of care. Executive Board 134th session. 2014 Jan. 23. 6p. 